\title{
Agreement between the Takeda UA-731 automatic blood pressure measuring device and the manual mercury sphygmomanometer: an assessment under field conditions in Newcastle upon Tyne, UK
}

\author{
Colin Cartwright, Nigel Unwin, Peter Stephenson
}

\begin{abstract}
Study objective - To assess agreement between two Takeda UA-731 automatic blood pressure measuring devices (referred to as machines $A$ and $B$ ) and two manual mercury sphygmomanometers.

Design - A ' $Y$ ' connector attached each Takeda UA-731 to a manual mercury sphygmomanometer. Simultaneous measurements were made on adult subjects.

Setting - A population based cardiovascular disease survey in Newcastle upon Tyne, UK.

Participants - Measurements on machine A were compared in 71 individuals (all women), and on machine $B$ in 75 individuals ( 9 men, 66 women). The age range of subjects was 28 to 76 years and median ages were 59 years for machine $A$ and 50 years for machine $B$.

Main results - Blood pressure (mmHg) ranged from 72 to 212 systolic and 44 to 102 diastolic. Both Takedas gave significantly lower readings than the manual devices for systolic and diastolic pressures: differences were mean (SD: 95\% CI) $3.7 \mathrm{mmHg}(6 \cdot 5: 2 \cdot 2,5 \cdot 2)$ for machine $A$ systolic, $2 \cdot 3 \mathrm{mmHg}(4 \cdot 5: 1 \cdot 3,3 \cdot 4)$ machine A diastolic; $1.8 \mathrm{mmHg}(6 \cdot 2: 0.4,3 \cdot 3)$ machine $B$ systolic, and $1.8(4 \cdot 4: 0 \cdot 8,2 \cdot 8)$ machine $B$ diastolic. On the British $\mathrm{Hy}-$ pertension Society criteria, machine $A$ was graded $C$ on systolic measurements and $B$ on diastolic; machine $B$ was graded $B$ on both systolic and diastolic measurements. Conclusions - The performance of these machines compares favourably with the Dinamap 8100, recently adopted for survey work by the Department of Health. The Takeda UA-731 looks promising for epidemiological survey work but before it can be fully recommended further evaluations are needed.
\end{abstract}

(f Epidemiol Community Health 1996;50:218-222)

Observer bias is one of the main sources of error in the indirect measurement of blood pressure (BP). ${ }^{1}$ The use of fully automated electronic BP monitors is one solution to the problem of observer bias, ${ }^{2}$ although even with fully automated machines differences caused by observer-subject interaction may still occur. ${ }^{3}$ As yet, however, automated devices have not superseded or even gained parity with the manual sphygmomanometer in a clinical or research setting. This is largely because the accuracy of those devices which have been tested is variable, ${ }^{4}$ and many devices remain untested.

The Takeda UA-731 blood pressure monitor has been available for several years, but has never been compared with a standard manual or random zero mercury sphygmomanometer. This study aimed to compare the agreement between two Takeda UA-731 blood pressure monitoring devices and standard manual mercury sphygmomanometers.

\section{Description and operation of the}

UA-731

The two Takeda UA-731 machines tested here were purchased in 1991 by the Department of Medicine at the University of Newcastle and used regularly, but not intensively (ie, up to about 20 measurements a week on each machine) over the two year period before this study was undertaken.

The Takeda UA-731 ${ }^{5}$ is battery powered, weighs $320 \mathrm{~g}$, and is $19 \mathrm{~cm} \times 4.2 \mathrm{~cm} \times 9 \mathrm{~cm}$ in size. The measurement range lies between $2 \mathrm{mmHg}$ and $280 \mathrm{mmHg}$ and accuracy of $\pm 3 \mathrm{mmHg}$ (or $2 \%$ of measured value) is claimed by the manufacturer. A micropump inflates the cuff when the start button is pressed. The system works by an oscillometric method The first pulse of systole (phase 1) causes a pulse pressure wave which is interpreted by the machine and shows as a readout for systolic pressure. Similarly, when the pulse wave disappears (phase 5) the machine gives the diastolic readout. The cuff automatically deflates by a constant air release valve system. The speed at which air is released during blood pressure determination (the exhaust rate) is adjustable by turning a screw on the rear of the device.

\section{Methods}

SUBJECTS

Subjects were participants in the Newcastle heart project (NHP), a cross sectional screening programme designed to estimate the prevalence of ischaemic heart disease, diabetes, and the 
risk markers for these conditions in the general population of Newcastle upon Tyne. The participants in the NHP were from an age and sex stratified random sample of 900 men and 900 women chosen from the family health services authority patient register. Most of the subjects in whom the Takeda machines were compared were female because at the time this study was carried out the male screening was drawing to a close. The measurements were made over a four month period.

Based on advice received from a medical statistician, the aim was to carry out simultaneous measurements on 100 individuals for each Takeda machine. Assuming a standard deviation (SD) on the difference between the Takeda and mercury sphygmomanometer measurements of $8 \mathrm{mmHg}$, the $95 \%$ confidence intervals $(95 \%)$ on the mean difference between the two sets of measurements would be $\pm 1.57 \mathrm{mmHg}$.

OBSERVATION AND BLOOD PRESSURE TESTING Two observers were trained to take blood pressure in the Newcastle heart project following the guidelines of the British Hypertension Society, as outlined in the booklet ${ }^{6}$ and video. ${ }^{7}$ Before starting to measure blood pressure in the heart project the observers used a two headed stethoscope to assess interobserver variation on 20 measurements. Agreement was within $4 \mathrm{mmHg}$ on all occasions. A record was kept of the observer for each measurement and each month the terminal digits for each observer were summarised and fed back to the observer. Examination of the distribution of terminal digits over the period of the study showed no evidence of a systematic preference in either observer. Every six months the training procedure using the booklet and video was repeated and interobserver variation assessed using the two headed stethoscope.

Two manual sphygmomanometers were used as the reference devices. They were the Accoson vertical type and were purchased specifically for use in the NHP. At each screening session it was checked that the mercury meniscus was at zero before inflation of the cuff, and the tubing was checked for wear. A plastic ' $\mathrm{Y}$ ' connector was used to link each automated machine to a manual sphygmomanometer. Thus, when the automated machine inflated and deflated the pressure in the cuff could be read on the manual machine at the same time. Blood pressure was measured in the right arm after the subject had been sitting quietly for at least three minutes. The arm rested in front of the subject on a pillow on a table. The height of the pillow and chair were adjusted so that the antecubital fossa was at the level of the mid sternum. The cuffs used were those supplied with the Takeda machines. The bladders of these cuffs are $23 \mathrm{~cm}$ in length and $12.5 \mathrm{~cm}$ wide. Before each measurement, any air remaining in the cuffs was squeezed out. The centre of the bladder was marked on the cuff and the cuff positioned so that the centre was over the brachial artery. The cuff was fitted snugly and the subject was instructed to remain relaxed and to keep the arm still during the measurement. The deflation rate for the automated machines was set at $2 \mathrm{mmHg}$ per second by adjusting the air exhaust valve. The inflation pressure was set at $160 \mathrm{mmHg}$ (if this is not sufficient, the Takeda automatically reinflates to $200 \mathrm{mmHg}$ or higher if necessary). The automated machine was placed so that it could not be seen by the observer. The start button was pressed and the stethoscope placed over the brachial artery. The manual mercury reading was taken and recorded, and then the Takeda measurement was read off and recorded. The diastolic measurement on the manual machine was taken at the disappearance of the Korotkow sounds (phase V).

\section{STATISTICAL ANALYSIS}

The data were entered onto a Database III file and analysed using SPSS (Statistical Package for Social Scientists). For each Takeda machine (referred to as machine A and machine B) the differences (manual minus Takeda) for systolic and diastolic blood pressure were calculated and plotted against the mean of the two measurements (Bland-Altman plots ${ }^{8}$ ). The 95\% confidence intervals were calculated for the mean difference. It was determined what proportion of the Takeda readings lay within 5, 10 , and $15 \mathrm{mmHg}$ of the manual mercury sphygmomanometer so that the machines could be graded following the recommendations of the British Hypertension Society. ${ }^{9}$ Linear regression equations were calculated for the difference between the Takeda and manual machine against the mean blood pressure level to determine if a linear trend existed in instrument differences with blood pressure level.

The mean difference between machine A and the manual device was compared to the mean difference between machine $B$ and the manual device using the independent samples $t$ test.

\section{Results}

Seventy one pairs of measurements in 71 different individuals (all women) were made on machine $A$ and 75 pairs of measurements in 75 different individuals (9 men and 66 women) on machine B. Most measurements were made by just one of the two observers: $57 / 71(80 \cdot 3 \%)$ on machine $\mathrm{A}$, and $60 / 75(80 \%)$ on machine $B$. The subjects were aged between 28 and 76 years, median age 59 years for machine $A$ and 50 years for machine $B$. The ranges of blood pressure measured (based on the manual mercury readings) for machine $A$ were: 46 to $102 \mathrm{mmHg}$ for diastolic $\mathrm{BP}$ and 88 to 212 for systolic BP. For machine B, the blood pressure ranged between 44 and $100 \mathrm{mmHg}$ for diastolic and 72 and $166 \mathrm{mmHg}$ for systolic BP. Both Takedas read significantly (all $\mathrm{p}$ values between $<0.0001$ and 0.012 ) lower at diastolic and systolic pressures than the standard mercury sphygmomanometers (table). The mean difference in the systolic reading in machine $\mathrm{A}$ was $3.7 \mathrm{mmHg}(95 \% \mathrm{CI} 2.2,5.2)$ and in 
Comparisons of measurements made simultaneously by the Takeda UA 731 devices and manual mercury sphygmomanometers

\begin{tabular}{|c|c|c|c|c|c|c|c|c|c|c|}
\hline \multirow[t]{2}{*}{ Device } & \multirow[t]{2}{*}{ No } & \multirow{2}{*}{$\begin{array}{l}\text { Meam } \\
(\mathrm{mmHg})\end{array}$} & \multirow[t]{2}{*}{$(S D)$} & \multicolumn{2}{|c|}{ Mean difference (SD) } & \multirow[t]{2}{*}{$95 \% C I$} & \multicolumn{3}{|c|}{ Readings (\%) } & \multirow[t]{2}{*}{ Grade } \\
\hline & & & & & & & $<5 m m H g$ & $<10 \mathrm{mmHg}$ & $<15 \mathrm{mmHg}$ & \\
\hline $\begin{array}{l}\text { Systolic: } \\
\text { Manual } \\
\text { Takeda A }\end{array}$ & $\begin{array}{l}71 \\
71\end{array}$ & $\begin{array}{l}127 \cdot 2 \\
123 \cdot 5\end{array}$ & $\begin{array}{l}(24 \cdot 2) \\
(25 \cdot 5)\end{array}$ & $3 \cdot 7$ & $(6 \cdot 5)$ & $2 \cdot 2-5 \cdot 2$ & $63 \cdot 4$ & $83 \cdot 1$ & $94 \cdot 4$ & $\mathrm{C}$ \\
\hline $\begin{array}{l}\text { Diastolic: } \\
\text { Manual } \\
\text { Takeda A }\end{array}$ & $\begin{array}{l}71 \\
71\end{array}$ & $\begin{array}{l}70 \cdot 5 \\
68 \cdot 1\end{array}$ & $\begin{array}{l}(11 \cdot 4) \\
(11 \cdot 1)\end{array}$ & $2 \cdot 3$ & $(4 \cdot 5)$ & $1 \cdot 3-3 \cdot 4$ & $76 \cdot 1$ & $94 \cdot 4$ & $98 \cdot 6$ & B \\
\hline $\begin{array}{l}\text { Systolic: } \\
\text { Manual } \\
\text { Takeda B }\end{array}$ & $\begin{array}{l}75 \\
75\end{array}$ & $\begin{array}{l}120 \cdot 8 \\
119 \cdot 0\end{array}$ & $\begin{array}{l}(20 \cdot 3) \\
(20 \cdot 1)\end{array}$ & $1 \cdot 8$ & $(6 \cdot 2)$ & $0 \cdot 4-3 \cdot 3$ & $66 \cdot 7$ & $88 \cdot 0$ & $97 \cdot 3$ & B \\
\hline $\begin{array}{l}\text { Diastolic: } \\
\text { Manual } \\
\text { Takeda B }\end{array}$ & $\begin{array}{l}75 \\
75\end{array}$ & $\begin{array}{l}69 \cdot 7 \\
67 \cdot 9\end{array}$ & $\begin{array}{l}(11 \cdot 0) \\
(11 \cdot 1)\end{array}$ & $1 \cdot 8$ & $(4 \cdot 4)$ & $0 \cdot 8-2 \cdot 8$ & $76 \cdot 0$ & $96 \cdot 0$ & 100 & B \\
\hline
\end{tabular}
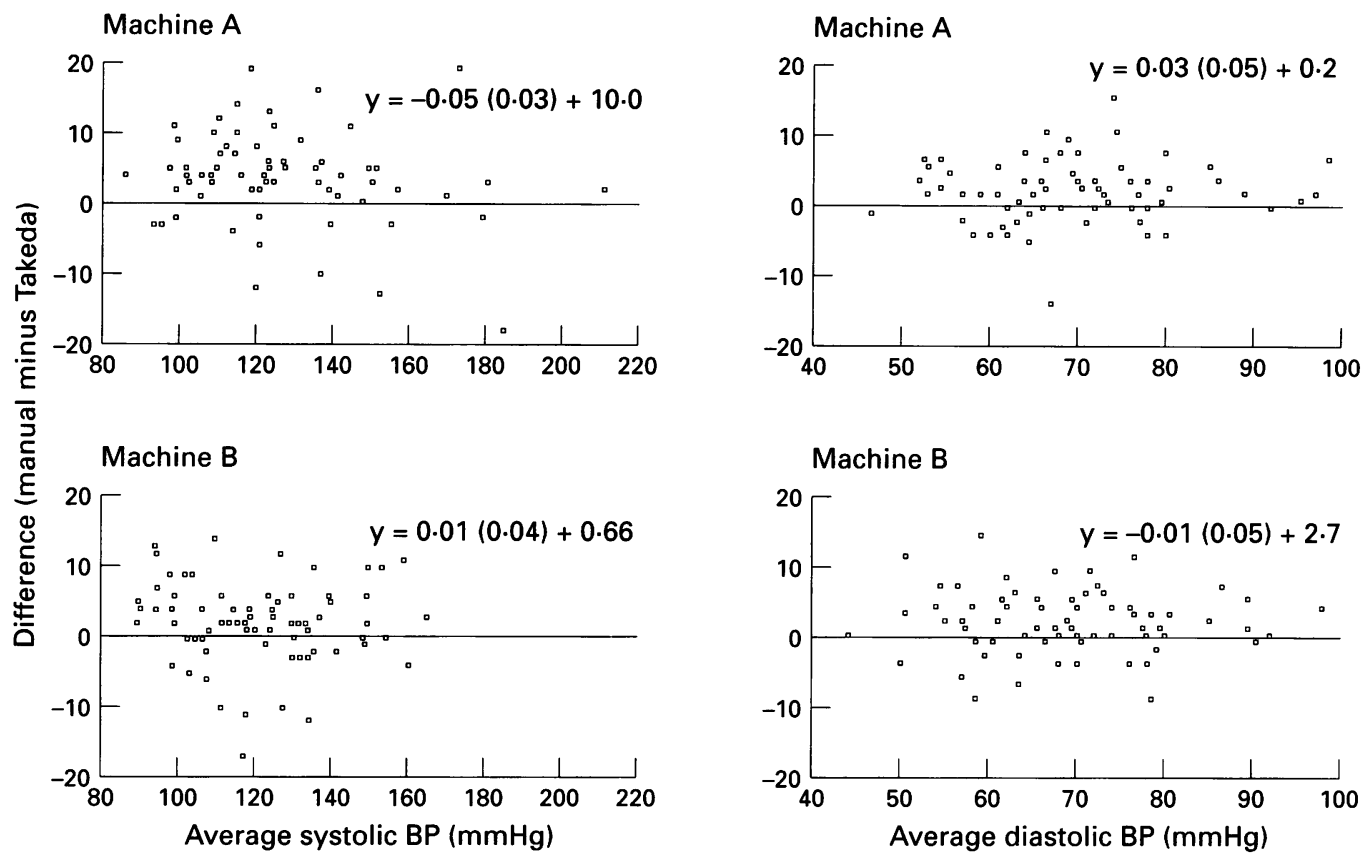

Plots of the difference (manual mercury minus Takeda UA 731 reading) against the mean (of manual and Takeda readings ( $A$ and $B)$ ). (Regression coefficients (SEM) plus constants are shown.)

machine $B$ it was $1.8 \mathrm{mmHg}(0.4,3.3)$. The difference between machines $\mathrm{A}$ and $\mathrm{B}$ was therefore $1.9 \mathrm{mmHg}(-0.2,+3.9, \mathrm{p}=0.08)$. The mean difference with manual measurement for the diastolic readings in machine $\mathrm{A}$ was $2.3 \mathrm{mmHg}$ and in machine $B$ it was $1.8 \mathrm{mmHg}$. The mean difference between machines $\mathrm{A}$ and $\mathrm{B}$ was $0.5 \mathrm{mmHg}(-0.9,+2.0$, $\mathrm{p}=0 \cdot 46)$.

The figure shows the difference between the manual mercury and the Takeda sphygmomanometers for systolic and diastolic measurements respectively, plotted against the mean of the manual and Takeda measurements (Bland-Altman plots). The percentages of pairs of measurements that were within 5,10 , and $15 \mathrm{mmHg}$ of each other are shown in the table. In neither machine $A$ or $B$, on systolic or diastolic readings, were $80 \%$ or more of the pairs of measurement within $5 \mathrm{mmHg}$ agreement. The systolic readings in machine A achieved only a $\mathrm{C}$ grade, and all the others $B$ grades. The regression equations for the difference against the mean blood pressure are shown next to the plots in the figure. There was no evidence of a significant linear trend in the size of the difference with blood pressure level in either machine in systolic or diastolic measurements.

\section{Discussion}

As far as we are aware this is the first comparison of the Takeda UA 731 automated blood pressure measuring device with a standard mercury sphygmomanometer. Because fewer subjects were available from the NHP than expected, the number of subjects on whom these machines were tested was less than originally planned. However, because the variances of the difference between the Takeda and manual mercury readings were less than allowed for in planning the study all the $95 \%$ CIs on the mean differences were within $\pm 1.5 \mathrm{mmHg}$.

The comparison between the Takeda UA 731 and the mercury sphygmomanometer was made by attaching the two machines together using a ' $\mathrm{Y}$ ' connector and taking simultaneous readings. This method was recommended in 
the British Hypertension Society protocol of $1990 .^{9}$ However, since then a revised version of this protocol ${ }^{10}$ has recommended that sequential, not simultaneous, measurements should be used because "the inflation-deflation characteristics of most devices do not permit simultaneous comparisons in the same arm". The Takeda UA 731 has an adjustable exhaust valve and so it was possible to adjust the deflation to facilitate simultaneous measurements. However, after measuring diastolic pressure the machine does deflate rapidly. It is perhaps surprising that this was not found to be a problem, and it was always possible to take a diastolic reading on the manual mercury sphygmomanometer. This may be partly because the Takeda machines tended to give lower readings than the manual devices, although, as is clear from the figure, on occasions the readings on the Takeda were higher than those made on the manual mercury. It is possible therefore that much of the difference between the Takeda and manual readings is not due to significant differences in the timing of the detection of phase 5 but to other forms of measurement error.

The accuracy of measuring devices after use is more relevant than the accuracy immediately after purchase, ${ }^{10}$ so it is of interest that the machines tested here had been in regular use for two years before this evaluation. A disadvantage of this, of course, is that we have no information on whether the accuracy of the machines changed over this two year period. We found that both Takeda machines evaluated here tended to read lower than the manual mercury devices: mean differences in systolic readings were 3.7 and $1.8 \mathrm{mmHg}$ for machines $\mathrm{A}$ and $\mathrm{B}$ respectively; and those in diastolic readings were 2.3 and $1.8 \mathrm{mmHg}$.

Although of a broad age range, most subjects in this study were women and the range of blood pressures was relatively limited - the highest diastolic pressure for example was $102 \mathrm{mmHg}$. There was no evidence of a change in the difference between the Takeda and the manual machines at different blood pressure levels (obviously we do not know if this would have been the case at blood pressure levels higher than those measured here). This suggests that for comparison with studies using the manual mercury sphygmomanometer it may be possible to subtract single correction factors for systolic and diastolic measurements respectively to measurements made with the Takeda UA-731. For this to be possible it would need to show that the differences for systolic and diastolic measurements are the same for different Takeda UA-731 machines. We compared two machines. There was little evidence that the two machines performed differently on diastolic readings. On the systolic readings, however, the difference was greater and approached statistical significance $(\mathrm{p}=$ $0 \cdot 08 ; 95 \%$ CI $-0 \cdot 2,+3 \cdot 9$ ).

The Department of Health has started to use an automated blood pressure measuring device, the Dinamap 8100, in the annual health survey for England. ${ }^{11}$ One of the reasons for choosing the Dinamap 8100 is that studies have sug- gested that it, or a very similar machine (Dinamap SX1846), measures blood pressure consistently over time. ${ }^{1213}$ This is clearly essential in survey work and an aspect of the performance of the Takeda machines evaluated here which we have not tested. In terms of comparability with a manual mercury sphygmomanometer, the Takeda machines tested here compare favourably to the Dinamap 8100 . A recent large study (1219 adults), commissioned by the Department of Health, compared the Dinamap 8100 with the manual mercury sphygmomanometer. ${ }^{14}$ Mean systolic pressure was higher when measured with the Dinamap $(7.9 \mathrm{mmHg} ; 95 \%$ CI $7.4,8.4)$, but mean diastolic levels were lower $(-1.8 \mathrm{mmHg}$; $95 \%$ CI $2 \cdot 3,-1 \cdot 3)$. The two Takeda machines tested here performed as well as the Dinamap 8100 on diastolic readings and better (ie, closer to the mercury sphygmomanometer readings) on systolic readings (the caveats must apply, of course, that the number of subjects tested in our study was less and the range of blood pressures narrower).

The British Hypertension Society has suggested grading criteria based on the proportion of paired measurements which fall within 5 , 10 , and $15 \mathrm{mmHg}$ of each other. ${ }^{8}$ Based on these criteria, neither machine achieved higher than a grade B on systolic or diastolic measurements (table). Machine A achieved a grade $\mathrm{C}$ on systolic measurements and as such would not have been recommended for clinical use according to the criteria of the British $\mathrm{Hy}$ pertension Society. However, both machines met the criteria specified by the American $\mathrm{Na}$ tional Standard for Electronic Automated Sphygmomanometers ${ }^{15}$ in which automated machines must achieve a mean difference of $\leqslant 5 \mathrm{mmHg}$ with an SD of no greater than $8 \mathrm{mmHg}$.

Automated machines of the type tested in this study offer several practical advantages to the measurement of blood pressure in epidemiological surveys. The main advantage is the elimination of some forms of observer bias which can occur with the manual mercury sphygmomanometer, notably digit preference and bias for and against certain levels of blood pressure. The Hawksley random zero sphygmomanometer is also a possible solution to these forms of bias and, interestingly, a recent review of the accuracy of the Hawksley random zero compared with the standard mercury sphygmomanometer, showed that the mean under ascertainment of diastolic and systolic blood pressure is of a similar order of magnitude to that found for the Takeda machines tested here. ${ }^{16}$ However, the Hawksley random zero machine disguises rather than eliminates digit preference and whether a standard mercury or a random zero sphygmomanometer is used observers need to be well trained in the interpretation of Korotkow sounds and reading from the mercury column scale. In addition, checks on the consistency between different observers in the interpretation of sounds and reading the scale need to be made; and a system of regular quality control on these aspects is essential. Training for and monitoring the use 
of an automated machine such as the Takeda is simpler, although as with a manual machine training is still required in the correct use of the machine, placement of the cuff, and positioning and preparation of the subject. The Takeda machine is also lighter, smaller, and easier to carry than a vertical manual sphygmomanometer or Hawksley random zero machine which would be an advantage in some survey situations.

In conclusion, we have assessed the agreement between two Takeda UA 731 machines and the standard mercury sphygmomanometer. Both Takeda machines were two years old at the time of testing, and had been used regularly over this period. The machines are light, compact, and easy to use. Although both machines read significantly lower than the manual mercury device, the differences were consistent over the range of blood pressures measured and they compare very favourably with a machine recently adopted for health surveys by the Department of Health, the Dinamap $8100 .^{14}$ Indeed, unlike the recent evaluation of the Dinamap, the Takeda machines meet the criteria of the American National Standard. ${ }^{15}$ The Takeda UA 731 looks promising for epidemiological survey work. However, before it can be recommended further evaluations are needed. Firstly, the machine should be tested on a broader range of blood pressures, particularly in the hypertensive range. Secondly, possible differences between machines should be further evaluated as our results suggest that a systematic mean difference between machine $A$ and machine $B$ in systolic readings may have existed. Finally, the consistency of the performance of the machines over time should be determined.

The authors wish to thank: Jane Harland for her advice and help in carrying out this study; Christopher Foy for statistical advice; and Surgicon Ltd (84 Wakefield Road, Brighouse, West Yorkshire) for the supply of technical information on the Takeda UA-731.

1 Bailey RH, Bauer JH. A review of common errors in the indirect measurement of blood pressure. Arch Intern Med 1993;153:2741-8

2 Stewart MJ, Padfield PL. Measurement of blood pressure in the technological age. Br Med Bull 1994;50:420-42.

3 Bruce NG, Cook DG, Shaper AG. Differences between observers in blood pressure measurement with an automatic oscillometric recorder. $\mathcal{F}$ Hypertens 1990;8(suppl): S11-S13.

4 O'Brien E, Atkins N, Mee F, O'Malley K. Comparative accuracy of six ambulatory devices according to blood accuracy of six ambulatory devices according

5 A\&D Company Ltd. UA-731 Digital blood pressure meter maintenance manual. A\&D Company Ltd, International maintenance manual. A\&D Company Ltd, International
Division, 1992. (Manual supplied by Surgicon Ltd, WakeDivision, 1992.

6 Petrie JC, O'Brien ET, Littler WA, de Swiet M, Padfield PL Dillon MJ. Blood pressure measurement, 2nd ed. London British Medical Journal, 1990.

7 Jamieson M, Petrie J, O'Brien E, Littler WA, de Sweit M Blood pressure measurement. Video for the British $\mathrm{Hy}$ pertension Society. London: British Medical Journal, 1990.

8 Bland AM, Altman DG. Statistical methods for assessing agreement between two methods of measurement. Lancet 1986;i:307-10.

9 O'Brien E, Petrie J, Littler W, de Sweit M, Padfield PL O'Malley $\mathrm{K}$, et al. The British Hypertension Society protocol for the evaluation of automated and semi-automated blood pressure measuring devices with special reference blood pressure measuring devices with special referen

10 to ambulatory systems. F Hypertens 1990;8:607-19. O'Brien E, Petrie J, Littler W, de Swiet M, Padfield PL,
Altman DG, et al. Short report: an outline of the revised British Hypertension Society protocol for the evaluation of blood pressure measuring devices. F Hypertens 1993 11:667-9.

11 White A, Nicolaas G, Foster K, Browne F, Carey S. Health survey for England, 1991. London: HMSO, 1993.

12 Ornstein S, Markert M, Litchfield L, Zemp L. Evaluation of the Dinamap blood pressure monitor in an ambulatory primary care setting. F Fam Pract 1988;26:517-21.

13 Whincup PH, Bruce NG, Cook DG, Shaper AG. The Dinamap 1846SX automated blood pressure recorder: comparison with the Hawksley random zero sphygmomanometer under field conditions. F Epidemiol Community Health 1992;46:164-9.

14 Bolling K. The Dinamap 8100 calibration study: a survey carried out by the Social Survey of the OPCS on behalf of the carried out by the Social Survey of the OPCS on beht
Department of Health. London: HMSO, 1994.

15 White WB, Berson AS, Robbins C, Jamieson MJ, Prisant LM, Roccella E, et al. National standard for measuring of resting and ambulatory blood pressures with automated sphygmomanometers. Hypertension 1993;21:504-9.

16 Mackie A, Whincup P, McKinnon M. Does the Hawksley random zero sphygmomanometer underestimate blood pressure, and by how much? $\mathcal{F}$ Hum Hypertens 1995;9 337-43. 